\title{
Gastric syphilis mimicking lymphoma
}

A 44-year-old Asian woman presented at hospital with a 3-week history of epigastric pain and early satiety. Physical examinations showed normal findings except for a minimally anemic appearance. Laboratory evaluation revealed a normal leukocyte count and serum protein level, a slight increase in the platelet count $\left(343 \times 10^{9} / \mathrm{L}\right)$ and C-reactive protein level $(13.9 \mathrm{mg} / \mathrm{L})$, and a small decrease in hemoglobin $(11.6 \mathrm{~g} / \mathrm{dL})$. Serologic tests for syphilis were positive for rapid plasmin reagin (RPR) and Treponema pallidum reagents. Serological findings for human immunodeficiency virus (HIV) were negative.

Upper gastrointestinal endoscopy revealed multiple, geographic, irregular ulcers from the gastric body to the pylorus $(\bullet$ Fig. 1a,b). The rapid urease test for Helicobacter pylori was negative. Ultrasonic gastroscopy revealed a slightly thickened gastric mucosa and interrupted submucosa associated with ulcerous lesions ( $\bullet$ Fig. 1 c). A computed tomographic scan of the abdomen with contrast showed non-specific mural thickening involving the gastric antrum ( $\bullet$ Fig. $1 d$ ). Gastric biopsies were performed and pathological analysis revealed tissue necrosis and an inflammatory infiltrate that was rich in lymphocytes, but evidence of malignancy was absent ( $\bullet$ Fig. 2a). Warthin-Starry staining revealed a mass of spirochetes that were infiltrating mucosal glands and vessels throughout the lamina propria ( $\bullet$ Fig. 2 b). The patient was treated with ceftriaxone ( $2 \mathrm{~g} /$ day, intravenously) because of having an allergy to penicillin. The patient's clinical complaints promptly disappeared, and endoscopic appearances subsequently improved.

Gastric syphilis is usually seen following secondary syphilis, and the rate of gastric involvement is only 1\% [1]. Endoscopy may reveal superficial ulcers with edema-
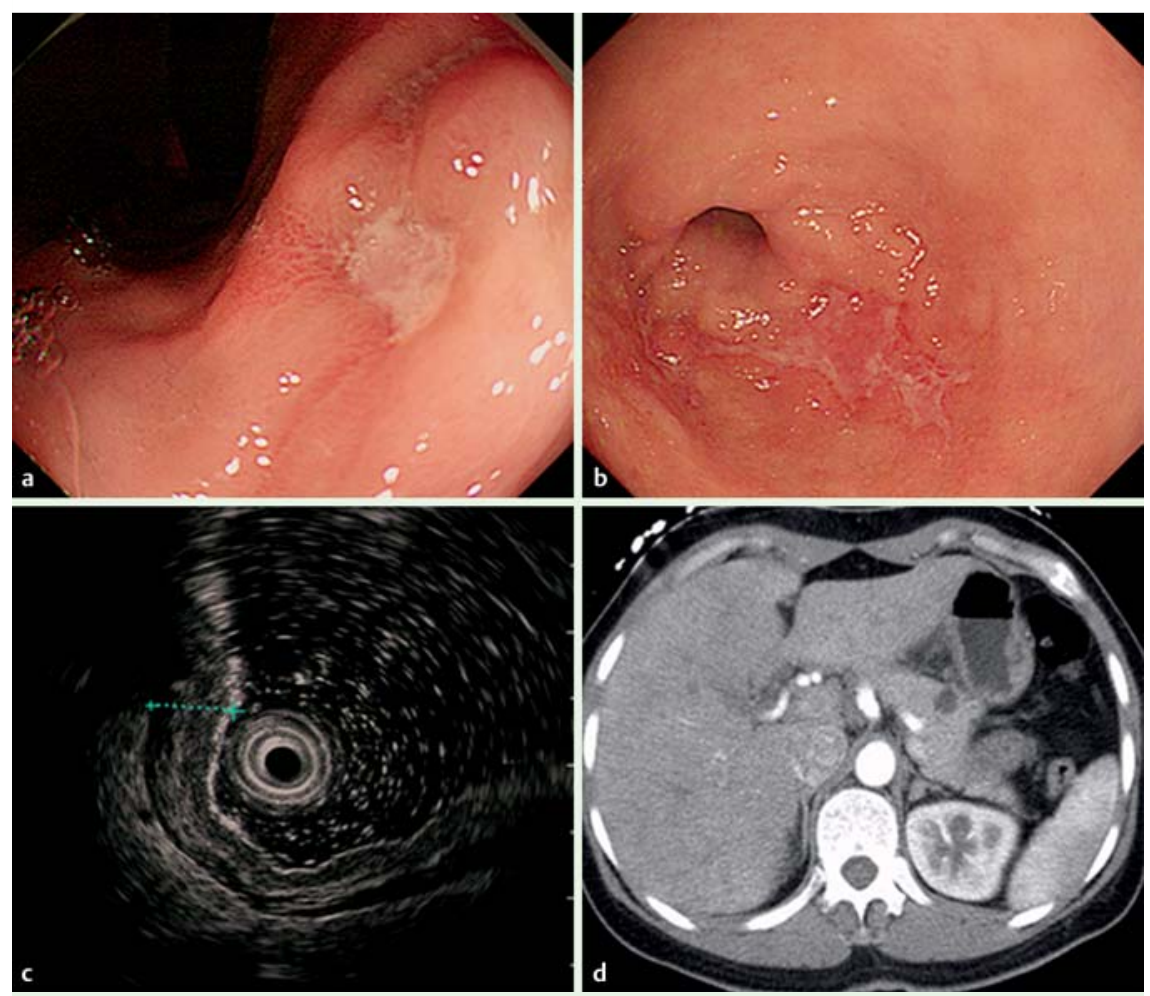

Fig. 1 Gastric syphilis in a 44-year-old woman before treatment. a, b Endoscopic appearances; c endoscopic ultrasound appearance; $\mathbf{d}$ computed tomography image.

tous mucosa, hyperplastic nodules, and hypertrophy of the rugae, which could be mistakenly considered as gastric carcinoma or lymphoma. In the histological diagnosis, dense lymphocytic infiltrates might easily be confused with lymphoma when lymphocytic phenotypes have not been identified [2]. In summary, when gastroscopic findings such as those described in this report are observed, gastric syphilis should be considered as an important diagnosis of suspicion in view of its nonspecific presentation.

Endoscopy_UCTN_Code_CCL_1AB_2AD_3AC

Competing interests: None
Yonghua Shen ${ }^{1}$, Ling $\mathrm{Nie}^{2}$, Ming Zhang ${ }^{1}$, Bei Tang', Zhengyan Qin ${ }^{1}$, Kui Meng ${ }^{2}$, Ying Lu ${ }^{1}$

${ }^{1}$ Department of Gastroenterology, Affiliated Drum Tower Hospital of Nanjing University, Medical School, Nanjing, People's Republic of China

2 Department of Pathology, Affiliated Drum Tower Hospital of Nanjing University, Medical School, Nanjing, People's Republic of China 


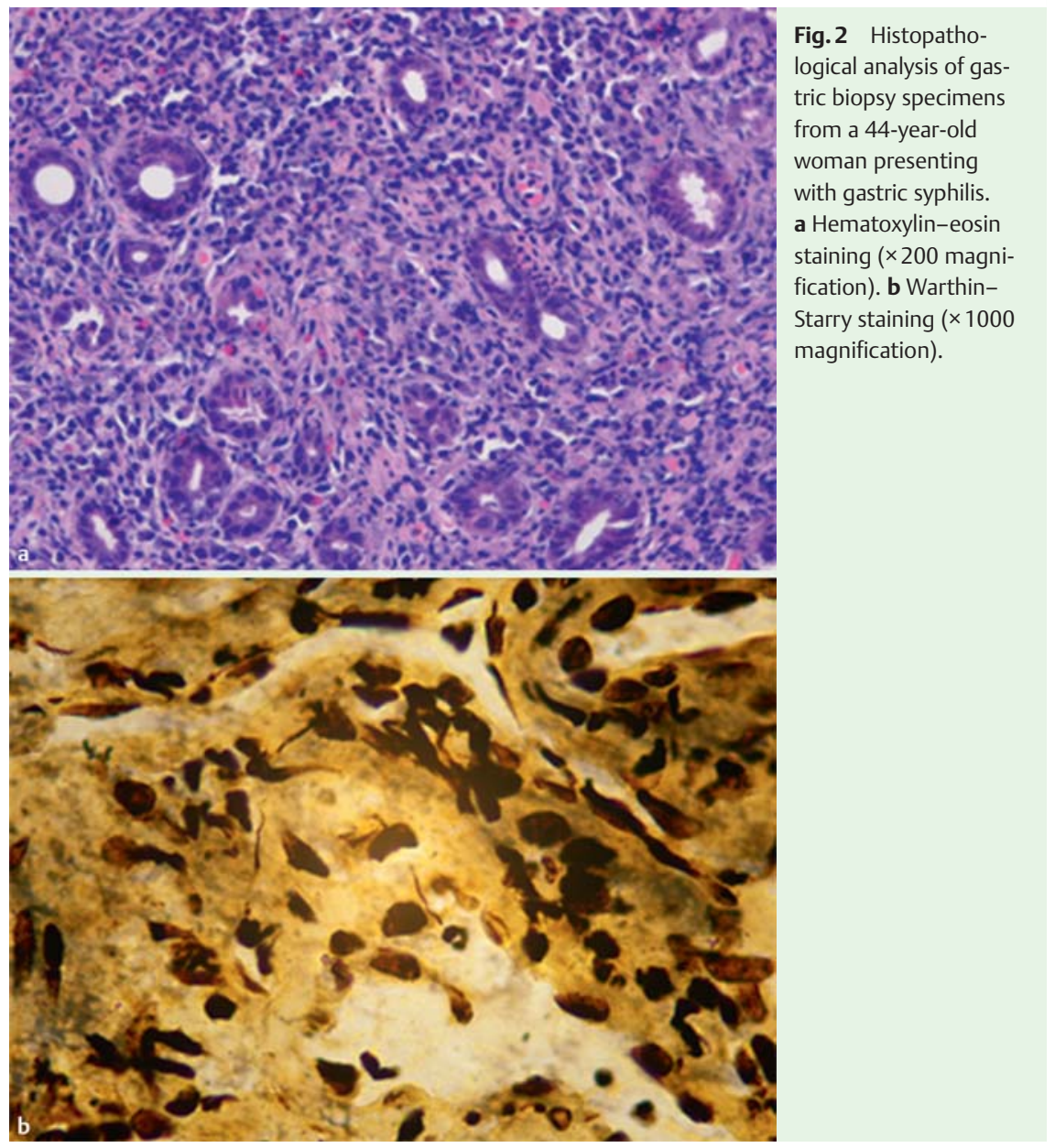

\section{References}

1 Atten MJ, Attar BM, Teopengco E et al. Gastric syphilis: a disease with multiple manifestations. Am J Gastroenterol 1994; 89: 2227 2229

2 Long BW, Johnston JH, Wetzel W et al. Gastric syphilis: endoscopic and histological features mimicking lymphoma. Am J Gastroenterol 1995; 90: 1504-1507

Bibliography

Dol http://dx.doi.org/

10.1055/s-0034-1391779

Endoscopy 2015; 47: E170-E171

(C) Georg Thieme Verlag KG

Stuttgart · New York

ISSN 0013-726X

\section{Corresponding author}

Ying Lu, MD

Department of Gastroenterology

Affiliated Drum Tower Hospital of Nanjing

University

Medical School

No. 321 Zhongshan Road

Nanjing 210008

People's Republic of China

Fax: +86-025-83105206

lying1999@126.com 